\title{
Multi-color 2D datamatrix codes with poorly readable colors
}

\author{
Authors: Urška Bogataj ${ }^{1}$, Tadeja Muck ${ }^{2}$, Branka Lozo ${ }^{3}$, Arjana Žitnik ${ }^{4}$ \\ ${ }^{1}$ Valkarton, Production of Corrugated Board and Packaging Inc., Logatec, Slovenia \\ ${ }^{2}$ University of Ljubljana, Faculty of Natural Sciences and Engineering, \\ Chair of Information and Graphic Technology, Ljubljana, Slovenia \\ ${ }^{3}$ University of Zagreb, Faculty of Graphic Arts, Zagreb, Croatia \\ ${ }^{4}$ University of Ljubljana, Faculty of Mathematics and Physics, Ljubljana, Slovenia
}

\begin{abstract}
Datamatrix code is a type of 2D codes that can encode much more data on the same or smaller area than the linear barcodes. This makes 2D codes usable for marking even very small items. 2D codes can be decoded by the readers in retails but also with the mobile phones equipped with camera and appropriate software. $2 \mathrm{D}$ codes can be depicted in different materials or printed on different printing substrates. The application area of the codes is broad, from magazines and newspapers to posters and packaging. Successful reading of 2D codes is possible if the code is printed in appropriate contrast between the printing ink and substrate, like black ink printed on white matt paper. Problems can occur if the code is printed in colors. The readability of 2D Datamatrix codes printed in cyan, magenta, yellow and black was studied. Yellow is proved to be poorly readable. In addition, the bi-colored and multi-colored 2D Datamatrix codes were studied. When four colors are used in creation of the 2D Datamatrix code, poorly readable elements, yellow codewords, may cause the reading failure. 2D Datamatix codes are capable to ensure good readability even if they contain a defined number of poorly readable codewords due to the Reed Solomon error-correction system. The aim of the study was to investigate the effect of using yellow printed, poorly readable, codewords in the multi-colored 2D Datamatrix code on the code readability.
\end{abstract}

Key words: 2D Datamatrix codes, readability, raster tone values, multi-colored codes, error-correction system, poorly readable codewords

\section{Introduction}

Automatic identification systems provide a fast and efficient identification of products. Among the most frequent are certainly the barcodes, which we encounter daily on the majority of retail products. Even though they enable a quicker identification of products their drawback is that they can encode only small amounts of (usually numerical) data and their size is not suitable for labeling small items. The concept of 2D codes is based on coding the data in both horizontal and vertical directions. Its development enabled the encoding of much larger quantity of

First received: 09.07.2010.

Accepted: 05.09.2010. numeric or alphanumeric data, up to 70 times compared to conventional barcodes, on a smaller area. This enabled the labeling and identification of even the smallest products. In addition, 2D codes are more and more used in different types of advertising and information management. 2D codes can encode the link to web pages and other types of information accessible by mobile phone capturing. Liu and coauthors (2008) describe the recognition of QR code with mobile phones. Laura Marriott (Mobile Marketing Association, n.d.) from Mobile Marketing Association describes her view to mobile marketing, physical goods purchasing, 2D codes and mobile coupons. Gao and coauthors (2007) explain the 2D code concepts, types and classifications, 2D code processing solutions 
for mobile application support and list the major technology players and applications in mobile commerce, Mobile com group (GS1, n.d.), supported by GS1, is an industry-wide initiative to enable businesses to give consumers access to product information via their mobile phones (Gao et. al, 2007). There are several standardized 2D codes, such as Datamatrix, QR Code, MaxiCode, Aztec Code, PDF417 Code and others, but mainly Datamatrix and QR Codes are used. Application of 2D codes varies from direct marking of small parts in metal, to computer and aviation industry; they are also used in medicine and pharmacy (Ostling Marking Sistems, 2009; Palmer, 2007). Moreover, the use of these codes is extended to areas such as advertising and marketing. 2D codes can be read by mobile phones equipped with a mobile phone camera and software for encoding 2D codes (Figure 1) (Anon, 2009; GS1, 2008).

$2 \mathrm{D}$ codes can be printed on different packaging and thus give to the customer additional information about the product or can be linked to the producer's web page or other content. The customer scans the code with a mobile phone camera, software in the mobile phone decodes the $2 \mathrm{D}$ code and the decoded message is visible on the mobile phone display (GS1, 2009; ScanLife, 2008).

$2 \mathrm{D}$ codes are usually printed ensuring maximum contrast, as black code printed on white substrate. This is due to the properties of reader devices which operate on the contrast-based principle. In advertising, design often demands the use of color other than black and white. This, however, might create problems in reading and decoding of the data.

The aim of the study was to define the limits of readability of cyan (C), magenta (M) and yellow (Y) colors used in the printing of 2D codes and to investigate the effect of using poorly readable codewords, yellow printed elements, in the multi-colored 2D Datamatrix code on the code readability.
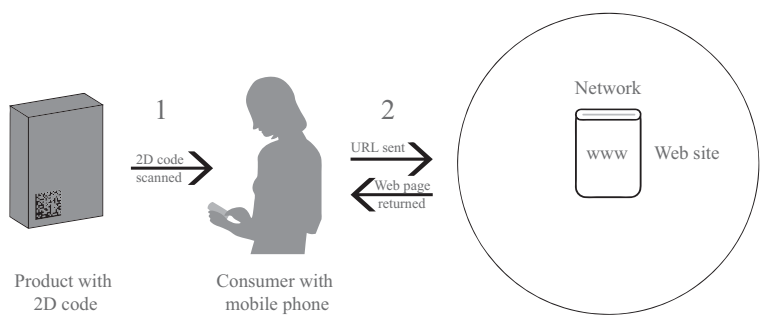

Figure 1. Identification process of $2 D$ code with mobile phone - 1. scanning of the code with mobile phone camera, 2. connection to the internet home page. (GS1, 2009)

\section{Theoretical part \\ Datamatrix Code}

Datamatrix code consists of black modules arranged in code matrix. Around the code there is a unique finder pattern used for orientation and determination of the symbol. Two adjacent sides, the left $\mathrm{y}$ and bottom $\mathrm{x}$ sides, forming the L boundary, are solid dark lines. They are used primarily to determine physical size, orientation and symbol distortion. The two opposite sides are made up of alternating dark and light modules. They are used primarily to define the cell structure of the symbol, but also can assist in determining of physical size and distortion (Figure 2) (ISO, 2006).

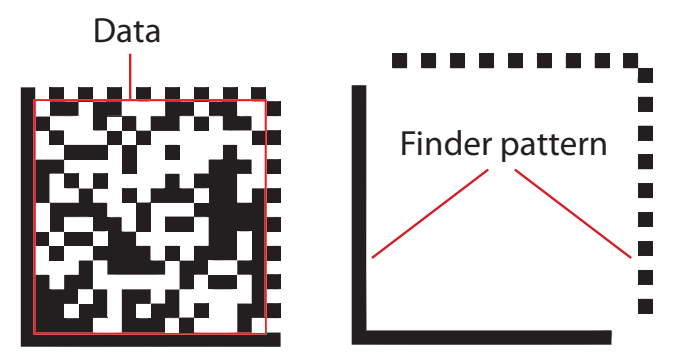

Figure 2. Datamatrix code (Kaywa Datamatrix., n.d.)

In an even number of rows and columns, the squareshaped $(10 \times 10$ to $144 \times 144)$ or rectangular-shaped $(8 \times 18$ to $16 \times 48)$ Datamatrix code can encode up to 2335 alphanumeric data characters, 15558 -bit byte data character or 3116 numerical data (ISO, 2006).

Datamatrix codes can be read even at only $20 \%$ of contrast between the printing ink and substrate according to the ISO/IEC 15415 (ISO, 2004). Datamatrix can encode data at only $1 / 10$ of space of the linear barcode (Williams, 2004).

\section{Code Readers}

Code readers operate on the principle of contrast between the code (printing ink) and the background (printing substrate). For code decoding various code readers are used and, in addition to readers, $2 \mathrm{D}$ codes can be decoded also with certain types of mobile phones. There are essentially three types (Formby, n.d.) of code readers:

\section{Camera-Based readers}

Camera-based readers are the newest type of code readers. This type of readers uses a small video camera to capture an image of the code. Sophisticated digital image processing techniques are then used to decode the code. Video cameras are equipped with the same CCD (Charge Coupled Device) technology as in a CCD code readers except that instead of having a single row of sensors, a video camera has hundreds of rows of sensors arranged in a two-dimensional array so that they can generate an image(Taltech, n.d.). Camera-based reader is the one used in capturing the codes with mobile phone camera (Figure 3 ).

\section{Laser readers}

Laser readers work on the principle of the red laser beams (wavelength around $650 \mathrm{~nm}$ ) emitted from the laser diode, which scan the code. The code reader con- 


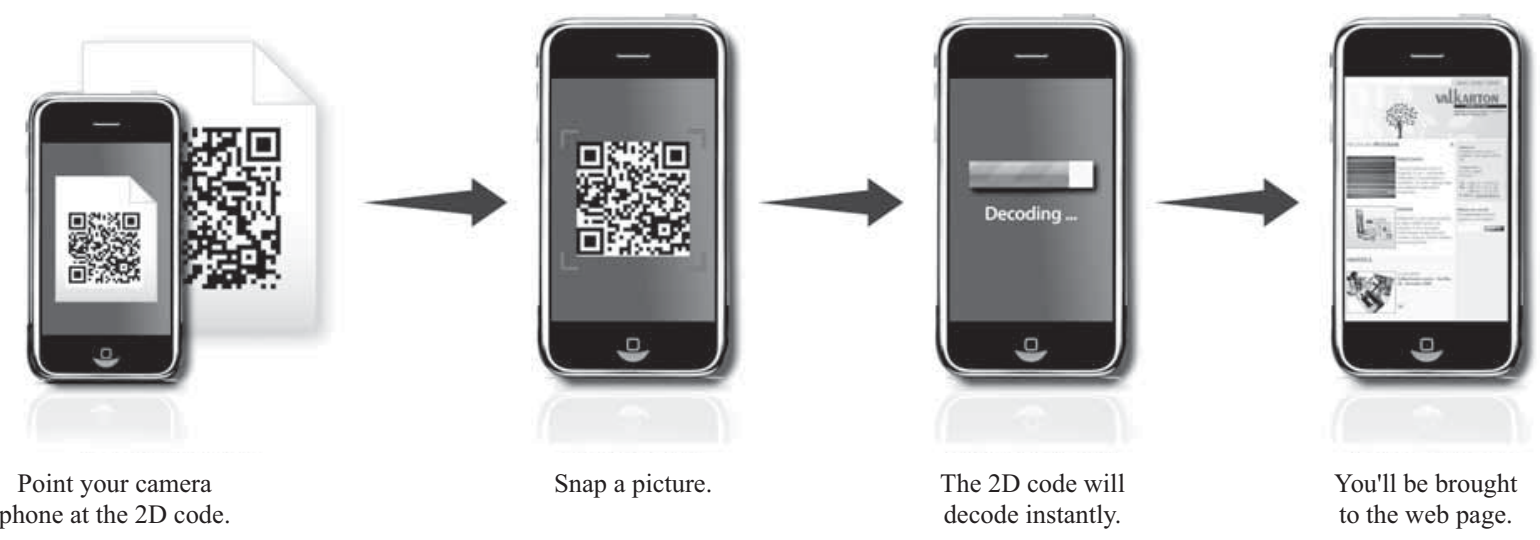

Figure 3. 2D code reading with mobile phone (QReate and track., $n . d$.)

verts the analog waveform to the digital one (A/D conversion). The code reader converts the signal combination of the printed and non-printed modules into the data according to the decoding rules (Keyence, n.d.).

\section{CCD readers}

CCD (Charge Coupled Device) readers use an array of hundreds of tiny light sensors lined up in a row positioned in the head of the reader. For providing good readability of codes the resolution between 1000 and 2000 pixels is desirable. Each sensor measures the intensity of the light immediately in front of it. CCD reader is measuring emitted ambient light from the code (Taltech, n.d.; World Intellectual Property Organization, 1994)

\section{Datamatrix Code Encoding}

The encoding of numerical, alphanumerical and other types (ASCII, kanji and kana symbols) of data in the

Table 1. Properties of information encoding into code symbol of different sizes. (ISO, 2006)

\begin{tabular}{|c|c|c|c|c|c|c|c|c|c|c|c|c|c|c|}
\hline \multicolumn{2}{|c|}{$\begin{array}{c}\text { Symbol } \\
\text { size }^{\mathrm{a}}\end{array}$} & \multicolumn{2}{|c|}{ Data region } & \multirow{2}{*}{$\begin{array}{l}\text { Mapping } \\
\text { matrix } \\
\text { size }\end{array}$} & \multicolumn{2}{|c|}{$\begin{array}{c}\text { Total } \\
\text { codewords }\end{array}$} & \multicolumn{2}{|c|}{$\begin{array}{l}\text { Reed- } \\
\text { Solomon } \\
\text { block }\end{array}$} & \multirow{2}{*}{$\begin{array}{l}\text { Inter- } \\
\text { leaved } \\
\text { blocks }\end{array}$} & \multicolumn{3}{|c|}{ Maximum data capacity } & \multirow{2}{*}{$\begin{array}{c}\% \text { of } \\
\text { codewords } \\
\text { used } \\
\text { for error } \\
\text { correction }\end{array}$} & \multirow{2}{*}{$\begin{array}{c}\text { Max. } \\
\text { correctable } \\
\text { codewords } \\
\text { Error / } \\
\text { erasure }\end{array}$} \\
\hline Row & Col & Size & No. & & Data & Error & Data & Error & & Num. & Alphanum. & Byte & & \\
\hline 10 & 10 & $8 \times 8$ & 1 & $8 \times 8$ & 3 & 5 & 3 & 5 & 1 & 6 & 3 & 1 & 62,5 & $2 / 0$ \\
\hline 12 & 12 & $10 \times 10$ & 1 & $10 \times 10$ & 5 & 7 & 5 & 7 & 1 & 10 & 6 & 3 & 58,3 & $3 / 0$ \\
\hline 14 & 14 & $12 \times 12$ & 1 & $12 \times 12$ & 8 & 10 & 8 & 10 & 1 & 16 & 10 & 6 & 55,6 & $5 / 7$ \\
\hline 16 & 16 & $14 \times 14$ & 1 & $14 \times 14$ & 12 & 12 & 12 & 12 & 1 & 24 & 16 & 10 & 50 & $6 / 9$ \\
\hline 18 & 18 & $16 \times 16$ & 1 & $16 \times 16$ & 18 & 14 & 18 & 14 & 1 & 36 & 25 & 16 & 43,8 & $7 / 11$ \\
\hline 20 & 20 & $18 \times 18$ & 1 & $18 \times 18$ & 22 & 18 & 22 & 18 & 1 & 44 & 31 & 20 & 45 & $9 / 15$ \\
\hline 22 & 22 & $20 \times 20$ & 1 & $20 \times 20$ & 30 & 20 & 30 & 20 & 1 & 60 & 43 & 28 & 40 & $10 / 17$ \\
\hline 24 & 24 & $22 \times 22$ & 1 & $22 \times 22$ & 36 & 24 & 36 & 24 & 1 & 72 & 52 & 34 & 40 & $12 / 21$ \\
\hline 26 & 26 & $24 \times 24$ & 1 & $24 \times 24$ & 44 & 28 & 44 & 28 & 1 & 88 & 64 & 42 & 38,9 & $14 / 25$ \\
\hline 32 & 32 & $14 \times 14$ & 4 & $28 \times 28$ & 62 & 36 & 62 & 36 & 1 & 124 & 91 & 60 & 36,7 & $18 / 33$ \\
\hline 36 & 36 & $16 \times 16$ & 4 & $32 \times 32$ & 86 & 42 & 86 & 42 & 1 & 172 & 127 & 84 & 32,8 & $21 / 39$ \\
\hline 40 & 40 & $18 \times 18$ & 4 & $36 \times 36$ & 114 & 48 & 114 & 48 & 1 & 228 & 169 & 112 & 29,6 & $24 / 45$ \\
\hline 44 & 44 & $20 \times 20$ & 4 & $40 \times 40$ & 144 & 56 & 144 & 56 & 1 & 288 & 214 & 142 & 28 & $28 / 53$ \\
\hline 48 & 48 & $22 \times 22$ & 4 & $44 \times 44$ & 174 & 68 & 174 & 68 & 1 & 348 & 259 & 172 & 28,1 & $34 / 65$ \\
\hline 52 & 52 & $24 \times 24$ & 4 & $48 \times 48$ & 204 & 84 & 102 & 42 & 2 & 408 & 304 & 202 & 29,2 & $42 / 78$ \\
\hline 64 & 64 & $14 \times 14$ & 16 & $56 \times 56$ & 280 & 112 & 140 & 56 & 2 & 560 & 418 & 277 & 28,6 & $56 / 106$ \\
\hline 72 & 72 & $16 \times 16$ & 16 & $64 \times 64$ & 368 & 144 & 92 & 36 & 4 & 736 & 550 & 365 & 28,1 & $72 / 132$ \\
\hline 80 & 80 & $18 \times 18$ & 16 & $72 \times 72$ & 456 & 192 & 114 & 48 & 4 & 912 & 682 & 453 & 29,6 & $96 / 180$ \\
\hline 88 & 88 & $20 \times 20$ & 16 & $80 \times 80$ & 576 & 224 & 144 & 56 & 4 & 1152 & 862 & 573 & 28 & $112 / 212$ \\
\hline 96 & 96 & $22 \times 22$ & 16 & $88 \times 88$ & 696 & 272 & 174 & 68 & 4 & 1392 & 1042 & 693 & 28,1 & $136 / 260$ \\
\hline 104 & 104 & $24 \times 24$ & 16 & $96 \times 96$ & 816 & 336 & 136 & 56 & 6 & 1632 & 1222 & 813 & 29,2 & $168 / 318$ \\
\hline 120 & 120 & $18 \times 18$ & 36 & $108 \times 108$ & 1050 & 408 & 175 & 68 & 6 & 2100 & 1573 & 1047 & 28 & $204 / 390$ \\
\hline 132 & 132 & $20 \times 20$ & 36 & $120 \times 120$ & 1304 & 496 & 163 & 62 & 8 & 2608 & 1954 & 1301 & 27,6 & $248 / 472$ \\
\hline \multirow{2}{*}{144} & \multirow{2}{*}{144} & \multirow{2}{*}{$22 \times 22$} & \multirow{2}{*}{36} & \multirow{2}{*}{$132 \times 132$} & \multirow{2}{*}{1558} & \multirow{2}{*}{620} & 156 & 62 & 8 & \multirow{2}{*}{3116} & \multirow{2}{*}{2335} & \multirow{2}{*}{1555} & \multirow{2}{*}{28,5} & \multirow{2}{*}{$310 / 590$} \\
\hline & & & & & & & 155 & 62 & 2 & & & & & \\
\hline
\end{tabular}

${ }^{a}$ Symbol size does not include quiet zones, i.e. module width on all four sides around the code. 
Datamatrix code conforms to ISO 16022 standard (ISO, 2006). The Datamatrix code basically consists of modules that represent binary 0 and 1 value, i.e. encoded data with finder pattern (Figure 3 ). The size of the Datamatrix code depends on the amount of data ranging from $10 \times 10$ to $144 \times 144$ modules. Actually, the minimum amount of information encoded is $8 \times$ 8 plus 2 modules: one on each side of the finder pattern. For larger quantities of encoded data, 4, 16 or 36 Datamatrix codes can be merged into one code. The amount of data that can be encoded into the matrix of each particular size is defined in standard (ISO, 2006) and shown in Table 1.

Datamatrix codes also include the Reed-Solomon error correction system that enables correct reading even if the code is partially damaged, torn or dirty.

The encoding process for Datamatrix code is divided into following steps:

- The size of the code is determined according to the type and amount of data (table 1);

- Data (numbers or letter) have to be changed into binary values ( 0 and 1$)$ using the convert table between ASCII, decimal and binary values;

- Binary values have to be entered into codeword as is shown in Figure 5;

- Codewords with binary values are entered into code matrix according to the defined rules (Figure 6);

- The binary value of 1 represents black and 0 value represents a white module;

- To complete the code, the finder pattern is added around the encoded data.

The basic form of data encoding is determined by the form of codeword, which consists of 8 binary bits distributed from position 1 to 8 as shown in the Figure 4 . In one such codeword we can encode two numbers or one letter.

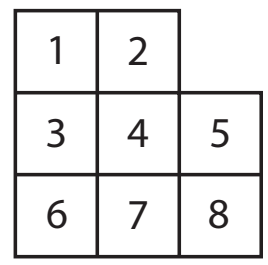

Figure 4. Codeword (ISO, 2006)

Positioning of codewords into the code matrix

After positioning of bytes into codewords, codewords have to be positioned into the code matrix. The positioning starts with the second codeword in the uppermost left corner. It is continued in a manner that all eight bits of each codeword are followed by a diagonal at $45^{\circ}$ as is shown in Figure 5. At some parts of the code, codewords have to be divided into more parts and placed into the code matrix according to the rules for codeword dividing defined as in the standard. At the codes with data region sizes $10 \times 10,14 \times 14,18 \times 18$ and $22 \times 22$ in the lower right corner 4 empty modules remain. Upper left and lower right are black the other two are white.

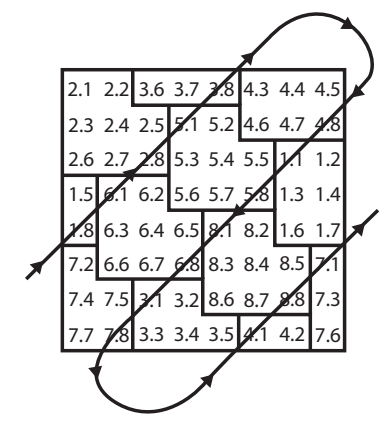

Figure 5. Positioning of codewords into the code matrix (ISO, 2006)

While positioning the codewords into the code matrix the codewords with encoded data are positioned first, followed by codewords for error correction. Thus, in Figure 6, data codewords are positioned in places 1, 2 and 3 and all the other codewords represent error correctors.
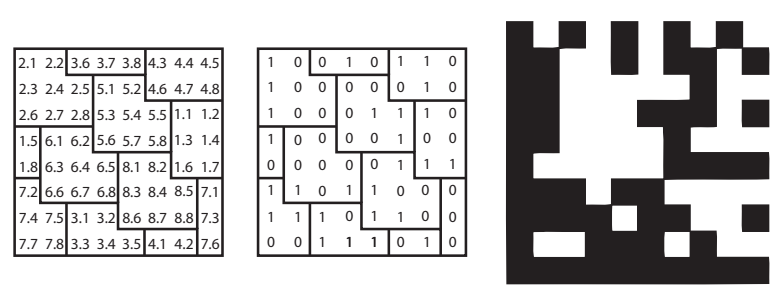

Figure 6. Positioning of codewords into the code matrix with $8 \times 8$ data region and final Datamatrix code with finder pattern (ISO, 2006)

Example of data encoding

According to the ASCII encoding procedure, to encode numerical values of two digits, 130 should be added to the number. Encoding of numbers $\gg 1 \ll, » 2 \ll, » 3 \ll, » 4 \ll$, $» 5 \ll$ and $» 6 \ll$ is presented below. ASCII encoding converts 6 characters to 3 bytes:

$» 12 \ll=12+130=142$

$» 34 \ll=34+130=164$

$» 56 \ll=56+130=186$

After a short calculation each "result" is represented with followed decimal numbers: 142, 164 and 186. The conversions according to ASCII, from decimal to binary values are shown in Table 2 (IBM, n.d.). The binary values are then put into codewords and inserted into the code matrix.

Table 2. Conversions from numbers to binary values
\begin{tabular}{|c|c|c|c|}
\hline $\begin{array}{c}\text { Pairs } \\
\text { of numbers }\end{array}$ & Adding 130 & $\begin{array}{c}\text { Result or } \\
\text { decimal value }\end{array}$ & $\begin{array}{c}\text { Binary } \\
\text { value }\end{array}$ \\
\hline$» 12 «$ & $12+130$ & 142 & 10001110 \\
\hline$» 34 \ll$ & $34+130$ & 164 & 10100100 \\
\hline$» 56 \ll$ & $56+130$ & 186 & 10111010 \\
\hline
\end{tabular}




\section{Reed-Solomon error correction}

To enable detection and correction of errors that arise when storing or reading data, the Datamatrix codes employ Reed-Solomon error-correction (Reed and Solomon, 1960). A Datamatrix code consists of data codewords and error correction codewords, which are computed from the data. It is known that adding $t$ error correcting codewords to data results in capability of correcting at most [ $t / 2]$ (codeword) errors when using a linear error-correcting code. This upper bound is achieved by the Reed-Solomon codes.

The description of the process of encoding data with Reed-Solomon codes for the $8 \times 8$ Datamatrix codes: all the arithmetic is done in the binary Galois field GF $\left(2^{8}\right)$ of order $\left(2^{8}\right)$, defined by the irreducible polynomial $f(x)=x^{8}+x^{5}+x^{3}+x^{2}+1$. That means that any codeword is represented as a polynomial of degree at most 7; addition is the usual addition of polynomials (reducing coefficients modulo 2) and multiplication is the usual multiplication of respective polynomials, followed by a reduction modulo $f(x)$ (and again reducing coefficients modulo 2). To produce five error correcting codewords from three data codewords, an appropriate irreducible generator polynomial $g(x)$ of degree 5 is used, namely $g(x)=x^{5}+62 x^{4}+111 x^{3}+15 x^{2}+48 x+228$ (the coefficients of $g$ are symbols from GF $\left(2^{8}\right)$, represented as decimal numbers). The data $s=\left(s_{1}, s_{2}, s_{3}\right)$ is then treated as the polynomial $s(x)=\left(s_{1} x^{2}+s_{2} x+s_{3}\right)$ of degree 2 with coefficients from GF $\left(2^{8}\right)$. The so-called check polynomial $c(x)$ is calculated and it is the remainder of the polynomial $s(x) \cdot x^{5}$ divided by the generator polynomial $g(x)$, i.e., there exists a polynomial $r(x)$ such that $s(x) \cdot x^{5}=r(x) g(x)+c(x)$. The coefficients of the check polynomial, which is of degree at most 4 , give the 5 error correcting codewords. This results in capability of correcting 2 corrupted codewords from any of the 8 Datamatrix codewords. A Datamatrix code is read correctly, if $g(x)$ divides $s(x)$ $\cdot x^{5}-c(x)$, where the polynomial $s(x)$ is formed from the first three codewords and $c(x)$ is formed from the remaining five codewords of the code. Otherwise a more sophisticated decoding algorithm has to be used, that determines the positions and values of the errors. The ISO 16022 standard (ISO, 2006) recommends and describes the Peterson-Gorenstein-Zierler decoding algorithm.

\section{Example of check-digit calculation}

Let us calculate the error correction codewords for the data »123456 « from the previous example. This data is represented with the decimal values 142, 164 and 186. The data polynomial is formed as $s(x)=142 x^{2}+164 x$ +186 . The check polynomial $s(x)=114 x^{4}+25 x^{3}+5 x^{2}$ $+8 x+102$ is calculated as the remainder of the polynomial $s(x) \cdot x^{5}$ divided by $g(x)$, the five error correct- ing codewords being $(114,25,5,88,102)$ in decimal notation, or (1110010, 00011001, 00000101, 1011000, $1100110)$ in binary notation. These codewords are then inserted into the code matrix as in Figure 7. All the arithmetic is done in the Galois field GF $\left(2^{8}\right)$ described above.

\section{Experimental part}

Previous research (Bogataj et. al, 2010) on the readability of $2 \mathrm{D}$ codes printed in several digital printing techniques and using different ink colors (CMYK) proved the possibility of printing $2 \mathrm{D}$ codes in different colors. The level of contrast of each individual color necessary for successful readability of the code was determined.

The creation of 2D Datamatrix codes in multiple colors offer additional options to advertisers and designers. Therefore, the readability of multi-color 2D Datamatrix codes was studied. The experimental part included:

1. The analysis of the readability of monochromatic codes (CMYK) printed in different raster tone values;

2. The analysis of the readability of codes with a finder pattern in black and data region in cyan, magenta or yellow;

3. The analysis of the readability of codes with a finder pattern in cyan, magenta or yellow and data region in black;

4. The analysis of the readability of multi-color codes.

\section{Materials and methods}

Code generating.

Code was generated using the Kaywa generator (Kaywa Datamatrix., n.d.). Numbers "123456" were encoded into the Datamatrix code. They were encoded into first three codewords according to the described procedure of data encoding.

\section{Staining the modules.}

Datamatrix codes were printed in four process inks: cyan, magenta, yellow and black in different raster tone values in $10 \%$ steps ranging from 10 to $100 \%$. Datamatrix codes with a finder pattern in cyan, magenta or yellow with a black data region were printed. Also, codes with a finder pattern in black and data region in cyan, magenta or yellow were printed.

The next step was the creation of multi-color codes. In the creation of this type of codes attention was paid to print no more than two codewords in yellow.

According to the standard the code with a data region of $8 \times 8$ modules (symbol size $10 \times 10$ ) provides two codewords that can be corrected (Table 1). It was assumed that yellow printed modules are poorly readable or not readable at all, while cyan, magenta and black printed modules are readable. In order to prove the ca- 
pability of correction the codes with three yellow codewords were also created. The hypothesis was that such codes will not be readable.

Printing.

The codes were printed on laser printer HP Color LaserJet 3550 with default settings and resolution $600 \times$ 600 dpi. The printing substrate was packaging cardboard Reno de Medici $230 \mathrm{~g} / \mathrm{m}^{2}$ (Italy).

Reading.

All samples were exposed to standard daylight in The Judge II (Gretag Macbeth) chamber and illuminated at $45^{\circ}$ angle. The readability of codes was detected using mobile phone Nokia N86 8MP with i-nigma software for decoding $2 \mathrm{D}$ codes. The readability of codes in real conditions at the workplace was determined also.

\section{Results and discussion}

The level of readability of each individual code color was determined according to the percentage of printed raster tone value (Figures 7 and 8).

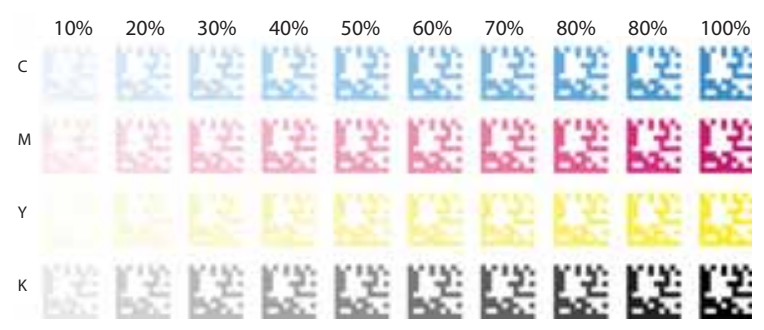

Figure 7. Cyan, magenta, yellow and black codes in different raster tone values.

Codes printed in cyan, magenta and black are readable at $30 \%$ raster tone value while the yellow code is readable at $70 \%$ raster tone value. In other words the yellow ink alone is poorly readable. Because of that fact and our intention to design codes with all four process inks, we assume that the readability of the code would only be possible with the support of the Reed-Solomon error corrector if the code consisted of not more than two code words in yellow.

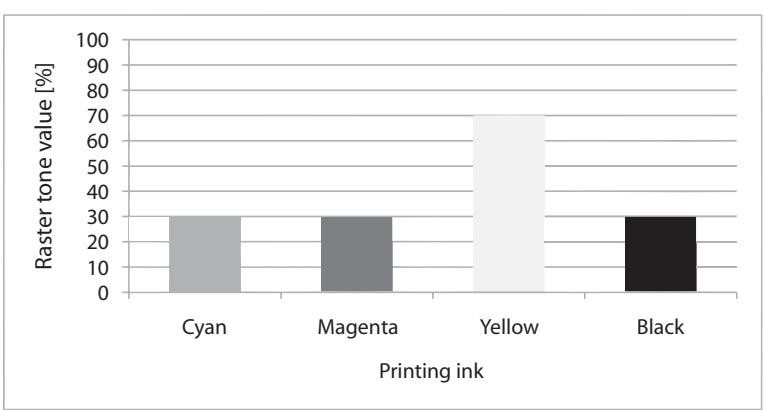

Figure 8. The lowest contrast of code that mobile phone can still read it
The analysis of readability of codes with a finder pattern in black and data region in cyan, magenta or yellow printed in $100 \%$ raster tone value was determined (Figure 9). The readability tests showed that Datamatrix codes with a finder pattern in black and data region in cyan and magenta were readable. The blackyellow combination was not readable. Even though the finder pattern was black and enough contrast was provided to read and to detect the orientation of the code, the mobile phone still could not read the code with yellow printed data.
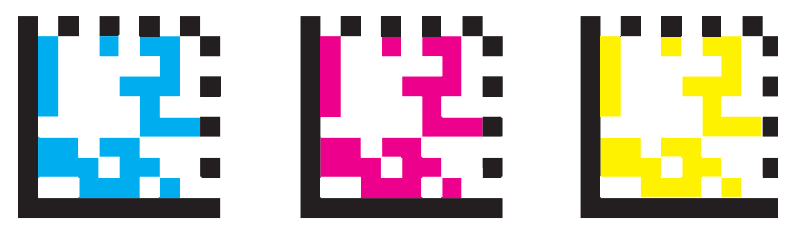

Figure 9. Codes with cyan, magenta and yellow data and black finder pattern

The analysis of readability of codes with a finder pattern in cyan, magenta or yellow and data region in black printed in $100 \%$ raster tone value was determined as well. Again, the cyan-black and magenta-black combinations were readable, while the yellow-black combination was not (Figure 10).
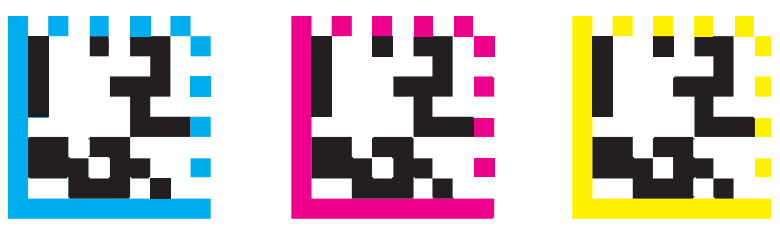

Figure 10. Codes with

black data and cyan, magenta

and yellow finder pattern

In the yellow-black combination the reader could not find the orientation of the code implying that the contrast between the finder pattern and the data region was too high.

\section{Analysis of multi-color codes}

2D Datamatrix codes consisting of codewords in all four colors were printed. Some codes had two and others had three yellow codewords (Figure 11). The experiment showed that the multi-color 2D Datamatrix codes with two yellow codewords were readable, while those with three yellow codewords were not. This was true for both readings, in chamber at standard daylight and in real conditions (Table 3). It is obvious that yellow is poorly readable, as only the codes with two yellow codewords were read successfully. The reading success of those codes is ascribed to the Reed-Solomon error correction system whose capability is to correct up to two errors in the code of this size $(8 \times 8$ data region, i.e. $10 \times 10$ code size). 

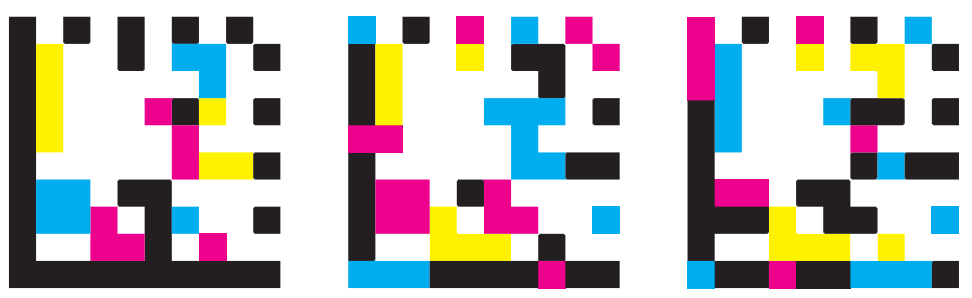

Three yellow codewords
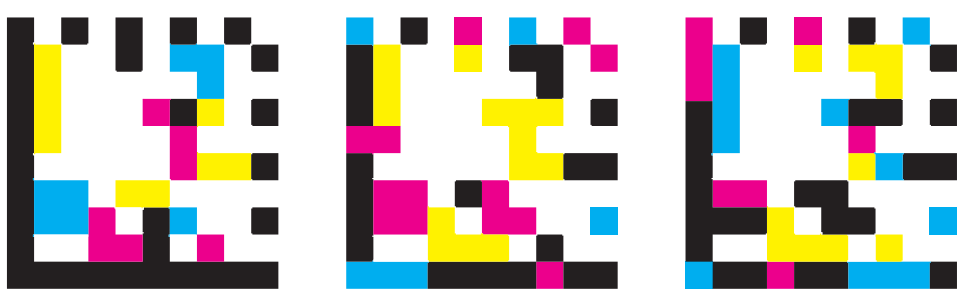

Three yellow codewords

Figure 11. Multi-color Datamatrix codes

Table 3. Multi-color Datamatrix readability results

\begin{tabular}{|c|c|c|}
\hline \multirow{2}{*}{$\begin{array}{c}\text { Number of } \\
\text { yellow } \\
\text { codewords }\end{array}$} & $\begin{array}{c}|c| \\
\text { Standard daylight } \\
\text { in chamber }\end{array}$ & $\begin{array}{c}\text { Real daylight } \\
\text { at workplace }\end{array}$ \\
\hline 2 & + & + \\
\hline 3 & - & - \\
\hline
\end{tabular}

\section{Conclusion}

The study has shown that 2D Datamatrix codes can easily be printed in different full colors or in different raster tone values. Regarding the readability, the colors behave differently, their level of readability depending on the raster tone value. The results of the experiment proved that cyan, magenta and black are readable at a $30 \%$ raster tone value, while yellow is readable at $70 \%$. In this respect, yellow proved to be poorly readable. In addition, the colors can be used in printing of code elements - finder pattern or data region. The codes printed in combinations of black and color in $100 \%$ raster tone value (black finder pattern and colored data region or vice versa) proved to be readable for cyan and magenta. When yellow is used either for the finder pattern (with black data region) or for data region (with black finder pattern) it is not readable at all. This implies that the contrast between the printing substrate and the printed code is not the only relevant factor for readability but the contrast between the code elements is relevant as well. It is also possible to produce the code using all four process colors, the multi-colored code. Due to the Reed-Solomon error correction option it is possible to accurately decode the data from multi-color codes if they contain up to two poorly readable elements (yellow codewords $)$ in an $8 \times 8$ data region $(10 \times 10$ code size). If the number of poorly readable codewords exceeds two, the entire code is not readable. We may conclude that multi-colored codes are suitable for advertising and marketing where some special color effect is attractive and codes are read with mobile phones that use camera-based readers.

\section{Acknowledgements}

The authors express their gratitude to the European Social Fund (»Operation part financed by the European Union, European Social Fund.«) and company Valkarton for their financial support and the Faculty of Natural Sciences and Engineering, University of Ljubljana for lab support as well as the Croatian National Scientific Project »Innovative printing materials $\ll$.

\section{References}

1. Anon. (2009) QR code and two dimensional bar codes, news, views and analysis. 2D Code. [Online] Avaliable from: http://2d-code.co.uk/ [Accessed 15 December 2009].

2. Bogataj, U., Muck, T., Bračko, S., Lozo, B. (2010) Readability of Processed Digital-Printed 2D Codes. Journal of Imaging Science and Technology 2010; in print

3. Formby, C. (n.d.) The Basics of Choosing a Barcode Reader. [Online] Avaliable from: http://ezinearticles.com/?The-Basics-Of-Choosing-A-BarcodeReader\&id=723146 [Accessed 29 December 2009].

4. Gao, ZJ., Prakash, L., Jagatesan, R. (2007) Understanding 2D-BarCode Technology and Applications in M-Commerce - Design and Implementation of A 2D Barcode Processing Solution. In: Compsac, 31st Annual International Computer Software and Applications Conference, 23-27 July 2007, Beijing, China. pp. 49-56.

5. GS1. Mobile Commerce: opportunities and challenges.(2008) GS1. A GS1 Mobile Com White Paper, February 2008 Edition. [Online] Avaliable from: http://www.gs1.org/docs/mobile/GS1_Mobile_Com_Whitepaper.pdf [Accessed 22 December 2009].

6. GS1. (2009) GS1 MobileCom Extended Packaging Pilot Handbook. Issue 1. [Online] GS1. Avaliable from: http://www.gs1.org/docs/mobile/GS1_Ex- 
tended_Packaging_Pilot_Handbook.pdf [accessed 22 December 2009].

7. GS1. GS1 MobileCom. (n.d.) [Online] Avaliable from: http://www.gs1.org/mobile [Accessed 12th December 2009].

8. IBM. (n.d.) ASCII, Decimal, Hexadecimal, Octal, and Binary Conversion Table. [Online] Avaliable from: http://publib.boulder.ibm.com/infocenter/systems/ index.jsp?topic=/com.ibm.aix.commadmn/doc/commadmndita/conversion_table.htm [Accessed 3 December 2009].

9. International Organization for Standardization (2004) ISO/IEC 15415. Information technology - Automatic identification and data capture techniques - Bar code print quality test specification - Two-dimensional symbols. Geneva, ISO

10. International Organization for Standardization (2006) ISO 16022. Information technology - Automatic identification and data capture techniques - Data Matrix bar code symbology specification. Geneva, ISO

11. Kaywa Datamatrix. (n.d.) Datamatrix Generator. [Online] Avaliable from: http://datamatrix.kaywa. com/ [Accessed 30 December 2009].

12. Keyence. (n.d.) Bar Code Reader Technical Guide. [Online] Avaliable from: http://www.keyence.com/ topics/barcode/bar_code/t_guide.php [Accessed 23 December 2009].

13. Liu, Y., Yang, J., Liu, M. (2008) Recognition of QR Code with Mobile Phones. The 2008 Chinese Control and Decision Conference, 2-4 July 2008 Yantai, China. pp. 203-206

14. Mobile Marketing Association. (n.d.) Mobile commerce: Coupons, 2D Codes and Purchases... Oh $m y$ !. [Online] Avaliable from: http://mmaglobal.com/articles/mobile-commerce-coupons2d-codes-and-purchasesoh-my [Accessed 12th December 2009].

15. Ostling Marking Sistems. Data Matrix 2D codes. (2009) [Online] Avaliable from: http://www.etchmark.co.uk/datamatrix/datamatrix.htm [Accessed 15 December 2009].

16. Palmer, R. C. (2007) The Barcode Book . Canada, Trafford.

17. QReate and track. (n.d.) Create your QR code. [Online] Avaliable from: http://app.qreateandtrack. com/\#/create/url [Acccessed 20 December 2009].

18. Reed, I. S., Solomon, G. (1960) Polynomial codes over certain finite fields. SIAM Journal of Applied Math. Vol. 8, pp. 300-304.

19. ScanLife. (2008) [Online] Avaliable from: http:// www.scanlife.com/atlantis/ [Accessed 5 January 2010].

20. Taltech. (n.d.) How a Barcode Reader Works. [Online] Avaliable from: http://www.taltech.com/ TALtech_web/resources/intro_to_bc/bcpwork.htm [Accessible 4 January 2009]
21. Williams, B. (2004) Understanding Barcoding. UK, PIRA

22. World Intellectual Property Organization. (1994) Two-dimensional, Portable CCD Reader. [Online] Avaliable from: http://www.wipo.int/pctdb/en/wo. jsp?IA=US1994005380\&DISPLAY=DESC [Accessed 4 January 2010]. 